This article deals with the problem of analyzing sets of ipsative variables using the common factor model. We demonstrate that the usual assumptions of the common factor model, especially the assumption of uncorrelated disturbances, are not appropriate for sets of ipsative variables. We develop a common factor model that takes into account the ipsative properties of such data and show how this model can be applied to any set of ipsative measures using the methods of confirmatory factor analysis. We then suggest that the application of this model may be useful in modeling the latent content of sets of rankings and other measures that have the ipsative property as a result of the measurement procedure. Finally, we apply the model to Kohn's measures of parental values, using sample data from the General Social Surveys.

\title{
The Factor Analysis of Ipsative Measures
}

\author{
DAVID J. JACKSON \\ National Institute of Mental Health \\ DUANE F. ALWIN \\ The University of Michigan
}

\section{INTRODUCTION}

Cattell (1944) initiated the use of the term "ipsative" (from the Latin ipse: he, himself) to refer to raw score transformations that center scores about the individual's mean. Ipsative scores were contrasted to the more common 'normative' scores, which are centered about the variable means. The ipsative transformation produces a set of scores with the property that the sum of the variables for each individual equals a constant.' Perhaps the most common use of scores produced by the ipsative transformation occurs in situations where it is desirable to remove mean differences in scores among individual cases or groups of

\footnotetext{
AUTHORS' NOTE: The authors wish to thank George W. Bohrnstedt and John $R$. Nessleroade for helpful suggestions on an early draft of this article and Cynthia $L$. Sipe for computational assistance.
} 
cases (e.g., Cunningham et al., 1977). Although, in the classical statement of the ipsative transformation, the constant is zero (because the individual's ipsative scores are deviations from his mean on the set of variables) it is now common to use the term "ipsative" to refer to any set of variables that sum to a constant for individual cases, regardless of the value of the constant (Horst, 1965: 290-291). The particular value of the constant is largely irrelevant.

It is important to note, as well, that sets of variables may occur which have the ipsative property, but not as a result of an ipsative transformation of nonipsative scores. For example, sets of variables produced from rankings and some Q-sorts have the ipsative property. The issues discussed in this article are particularly relevant to such sets of variables.

Numerous warnings exist in the psychometric literature against the application of factor analysis to sets of variables with the ipsative property (e.g. Cattell, 1952; Guilford, 1954; Tucker, 1956; Horst, 1965), but these warnings are not always heeded. In this paper we will underscore the basis for such warnings by showing that the assumptions of the common factor model, as usually stated, are inappropriate for sets of variables with ipsative properties. This is demonstrated within the framework of the development of a common factor model that can be applied to any set of variables with the ipsative property. We will show that a common factor model is defined for a set of variables that have undergone an ipsative transformation if a common factor model holds for the preipsative set, and we will show that for sets of ipsative variables that do not result from and ipsative transformation, the same model may be used under a set of assumptions regarding the response process. Finally, we will illustrate the usefulness of the model for the latter case by applying it to sample data on a set of ipsative measures of parental values.

\section{THE IPSATIVE TRANSFORMATION}

Let $y$ be a $(p \times 1)$ vector of random variables observable in some known population. The only constraints on $y$ are that the 
variables have a common metric and that the y covariance structure is positive-definite. The general form of the ipsative transformation of $y$ is as follows:

$$
x=y-1 w
$$

where $x$ is $a(p \times 1)$ vector of variables with the ipsative property, 1 is a $(p \times 1)$ unit vector and $w$ is a scalar. In the general case, $w$ equals $p^{-1}\left(1^{\prime} y-c\right)$, where $c$ is the constant to which the scores of each individual sum. This transformation, then, produces a set of variables that sum to $c$ for each individual; however, the value of the constant is irrelevant. ${ }^{2}$ As a matter of convenience we may set $c$ equal to zero, so that $w=p^{-1} 1^{\prime} y$.

Equation 1 may be rewritten as

$$
\begin{aligned}
x & =y-1 p^{-1} 1^{\prime} y \\
& =y-p^{-1} 11^{\prime} y \\
& =\left(I-p^{-1} U\right) y
\end{aligned}
$$

where $\mathrm{U}=11^{\prime}$ (a $\mathrm{p} \times \mathrm{p}$ matrix of unities). The ipsative transformation matrix in equation $2 c,\left(I-p^{-1} U\right)$, is a symmetric idempotent matrix with a rank of p- 1 (see Horst, 1965: 288-289; Harris, 1953: 54).

Let the ipsative transformation be represented by $A=(I-$ $p^{-1} U$ ), so that $x=A y$. The covariance matrix for $x$ may be expressed using this notation as

$$
\Sigma_{\mathrm{x}}=\mathbf{A} \Sigma_{\mathrm{y}} \mathrm{A}
$$

where $\Sigma_{y}$ is the covariance matrix of the preipsative variables. As noted, A has a rank of $p-1$, so this constitutes a singular transformation of $\Sigma_{y}$. Consequently, $\Sigma_{x}$ is singular and cannot be analyzed in the same manner that is possible for $\Sigma_{y}$.

The ipsative covariance matrix, $\Sigma_{x}$, has a number of interesting properties (see Clemans, 1956):

(1) The rows (or columns) of $\Sigma_{x}$ sum to zero.

(2) The sum of the covariances of a set of ipsative variables with a criterion variable is zero.

(3) As p increases, $\Sigma_{x}$ approaches $\Sigma_{y}{ }^{3}$ 
Because the ipsative covariance matrix is not positive-definite, it may not be feasible to subject $\Sigma_{x}$ to routine factor analysis. ${ }^{4}$ It is possible, however, to delete one row and column associated with an arbitrarily selected variable in order to obtain a nonsingular matrix, but the routine factor analysis of this submatrix does not provide a solution to the ipsative problem.

\section{A COMMON FACTOR MODEL FOR IPSATIVE MEASURES}

In this section of the article we will develop a common factor model that can be applied to ipsative measures. As we pointed out earlier, our primary interest here is in data that have not been "ipsatized" by an ipsative transformation, but which have the ipsative property as a result of the measurement method. In order to develop a factor model that can be applied in this situation, we first must develop a factor model for variables that have undergone an ipsative transformation. To do this we assume that a factor model exists for the set of preipsative variables. Then, by transforming the coefficient matrices of this model, we may obtain the coefficient matrices of the ipsative factor model. We turn now to the development of this model.

Let

$$
y=\wedge_{y} \quad \xi+\epsilon
$$

be the population common factor model for the set of measured nonipsative variables, $y$, where $\wedge_{y}$ is the factor pattern coefficient matrix, $\xi$ is a $(k \times 1)$ vector of common factors, and $\epsilon$ is a $(p \times 1)$ vector of disturbances (see Lawley and Maxwell, 1971). For our purposes the properties of this model are as follows:

(1) $\mathrm{E}(\mathrm{y})=0$

(2) $\mathrm{E}(\xi)=0$

(3) $\mathrm{E}(\epsilon)=0$

(4) $\mathrm{E}\left(\xi^{\prime}\right)=0$

(5) $E\left(\xi \xi^{\prime}\right)=\Phi_{y}$

(6) $\mathrm{E}\left(\epsilon \epsilon^{\prime}\right)=\Psi_{y}^{2}$

For convenience we have treated all variables, latent and manifest, as centered, and we assume that $\xi$ and $\epsilon$ are statistically 
independent. The matrices $\Phi_{\mathrm{y}}$ and $\Psi_{\mathrm{y}}^{2}$ are the covariance matrices for the factors and disturbances respectively. Here we assume that $\Psi_{y}^{2}$ is a positive-definite diagonal matrix, but in general the assumption that $\Psi_{y}^{2}$ is diagonal is not necessary. The covariance structure of $y$ may be written in terms of the model as:

$$
E\left(y^{\prime}\right)=\Sigma_{y}=\wedge_{y} \Phi_{y} \wedge_{y}^{\prime}+\Psi_{y}^{2}
$$

Given the above model and knowledge of the properties of the ipsative transformation, we may obtain the ipsative common factor model by substituting equation 4 for $y$ in equation $2 b$, as follows:

$$
\begin{aligned}
\mathbf{x} & =\left(\wedge_{y} \xi+\epsilon\right)-1 p^{-1} l^{\prime}\left(\wedge_{y} \xi+\epsilon\right) \\
& =\left(\wedge_{y} \xi+\epsilon\right)-1\left(\overline{\lambda^{\prime} \xi}+\bar{\epsilon}\right)
\end{aligned}
$$

where $\bar{\lambda}$ is a $(k \times 1)$ vector of the average values of the coefficients in the columns of $\Lambda_{y}$, and $\bar{\epsilon}$ is the average value of the residual disturbances on the model for y (see equation 4 ). We may rearrange equation $6 \mathrm{~b}$ as follows:

$$
\mathbf{x}=\left(\wedge_{y}-1 \bar{\lambda}^{\prime}\right) \xi+\epsilon-1 \bar{\epsilon}
$$

and because $(\epsilon-1 \bar{\epsilon})=A \epsilon$, equation $6 \mathrm{c}$ may be rewritten as

$$
x=\left(\wedge_{y}-1 \overline{\lambda^{\prime}}\right) \xi+A \epsilon
$$

This is the common factor representation for a set of ipsative variables which have the ipsative property as a result of an ipsative transformation, given knowledge that a common factor model for the preipsative variables exists.

Note that the primary differences in the factor models for the ipsative and preipsative variables are their coefficient matrices. The factor pattern coefficient matrix for $\mathrm{y}$ is $\Lambda_{y}$, while the factor pattern matrix for $x$ is $\left(\Lambda_{y}-1 \bar{\lambda}\right)$. As a result of the ipsative transformation, then, the factor pattern coefficients in the model for $\mathrm{x}$ are deviations of the factor pattern coefficients in $\wedge_{y}$ from the average factor pattern coefficients in the columns 
of $\wedge_{y}$. The coefficient matrix relating the disturbances to the $\mathrm{x}$ vector is $\mathrm{A}$ rather than $\mathrm{I}$, as is implicitly the case for $\mathrm{y}$. Since $\mathrm{A}$ is nondiagonal, the ipsative transformation affects the disturbances by correlating them in a systematic fashion.

The common factor model for $\mathbf{x}$, then, can be written as follows:

$$
\mathbf{x}=\left[\Lambda_{\mathbf{x}} \mid \mathbf{A}\right]\left[\begin{array}{l}
\xi \\
\epsilon
\end{array}\right]
$$

where $\Lambda_{\mathrm{x}}=\left(\Lambda_{\mathrm{y}}-1 \bar{\lambda}^{\prime}\right)$ and $\mathrm{A}=\left(\mathrm{I}-\mathrm{p}^{-1} \mathrm{U}\right)$. In addition to the ipsative property of $\mathrm{x}$, the properties of this model are as follows:

(1) $\mathrm{E}(\mathrm{x})=0$

(2) $\mathrm{E}(\xi)=0$

(3) $\mathrm{E}(\epsilon)=0$

(4) $\mathrm{E}\left(\xi \epsilon^{\prime}\right)=0$

(5) $\mathrm{E}\left(\xi \xi^{\prime}\right)=\Phi_{\mathrm{y}}$

(6) $\mathrm{E}\left(\mathrm{A} \epsilon \epsilon^{\prime} \mathrm{A}\right)=\mathrm{A} \Psi_{\mathrm{y}}^{2} \mathrm{~A}=\Psi_{\mathrm{x}}^{2}$

The covariance structure for $\mathrm{x}$ may then be written as:

$$
\mathrm{E}\left(\mathrm{xx}^{\prime}\right)=\Sigma_{\mathrm{x}}=\Lambda_{\mathrm{x}} \Phi_{\mathrm{y}} \Lambda_{\mathrm{x}}^{\prime}+\mathrm{A} \Psi_{\mathrm{y}}^{2} \mathrm{~A}
$$

The above factor model for $\mathrm{x}$ follows from the assumptions posed from the beginning, that (a) $x=A y$ and (b) $y=\Lambda_{y} \xi+\epsilon$. Note that by the transformation of $y$ into $x$, the disturbances on the factor model for $\mathrm{x}$ become correlated. As indicated above, the covariance structure of the disturbances in the model for $\mathrm{x}$ is $\Psi_{\mathrm{x}}^{2}=\mathrm{A} \Psi_{\mathrm{y}}^{2} \mathrm{~A}$. The diagonal elements of this matrix are $\psi_{\mathrm{x}_{\mathrm{i}}}^{2}=\left(1-2 \mathrm{p}^{-1}\right) \psi_{\mathrm{y}_{\mathrm{i}}}^{2}+\mathrm{p}^{-1} \bar{\psi}_{\mathrm{y}}^{2}$ and the off-diagonal elements are $\psi_{\mathbf{x}_{\mathrm{ij}}}=-\mathrm{p}^{-1}\left(\psi_{\mathrm{y}_{\mathrm{i}}}^{2}+\psi_{\mathrm{y}_{\mathrm{j}}}^{2}-\bar{\psi}_{\mathrm{y}}^{2}\right)$, where $\psi_{\mathrm{y}_{\mathrm{i}}}^{2}$ and $\psi_{\mathrm{y}_{\mathrm{j}}}^{2}$ are the $\mathrm{i}^{\text {th }}$ and $j^{\text {th }}$ elements in the diagonal of $\Psi_{y}^{2}$ and $\bar{\psi}_{y}^{2}$ is the average of the diagonal elements of $\Psi_{y}^{2}$. 


\section{AN ILLUSTRATION}

Consider the following $\mathrm{p}=5, \mathrm{k}=1$ factor model for some known population:

$$
\begin{aligned}
y & =\left[\begin{array}{l}
1.0 \\
1.1 \\
1.2 \\
1.3 \\
1.4
\end{array}\right] \xi+\epsilon \\
\Phi_{y} & =[2.0]
\end{aligned}
$$

Diag. $\Psi_{\mathrm{y}}^{2}=\left[\begin{array}{lllll}.90 & 1.2 & 1.0 & .80 & .60\end{array}\right]$

The population covariance structure for $y$ given this model is:

$$
\Sigma_{y}=\left[\begin{array}{lllll}
2.90 & 2.20 & 2.40 & 2.60 & 2.80 \\
2.20 & 3.62 & 2.64 & 2.86 & 3.08 \\
2.40 & 2.64 & 3.88 & 3.12 & 3.36 \\
2.60 & 2.86 & 3.12 & 4.18 & 3.64 \\
2.80 & 3.08 & 3.36 & 3.64 & 4.52
\end{array}\right]
$$

For $\mathrm{p}=5$ the ipsative transformation matrix, as defined in equation $2 \mathrm{c}$, is:

$$
\mathrm{A}=\left[\begin{array}{rrrrr}
.80 & -.20 & -.20 & -.20 & -.20 \\
-.20 & .80 & -.20 & -.20 & -.20 \\
-.20 & -.20 & .80 & -.20 & -.20 \\
-.20 & -.20 & -.20 & .80 & -.20 \\
-.20 & -.20 & -.20 & -.20 & .80
\end{array}\right]
$$

The ipsative transformation of $\Sigma_{\mathrm{y}}$ into $\Sigma_{\mathrm{x}}$ is as follows:

$$
\Sigma_{\mathrm{x}}=\left[\begin{array}{rrrrr}
.80 & -.20 & -.20 & -.20 & -.20 \\
-.20 & .92 & -.26 & -.24 & -.22 \\
-.20 & -.26 & .78 & -.18 & -.14 \\
-.20 & -.24 & -.18 & .68 & -.06 \\
-.20 & -.22 & -.14 & -.06 & .62
\end{array}\right]
$$


Finally, the ipsative factor coefficent matrix is:

$$
\Lambda_{\mathrm{x}}=\left(\Lambda_{\mathrm{y}}-1 \bar{\lambda}^{\prime}\right)=\left[\begin{array}{r}
-.20 \\
-.10 \\
.00 \\
.10 \\
.20
\end{array}\right]
$$

It can be shown that $\Lambda_{\mathrm{x}} \Phi_{\mathrm{y}} \Lambda_{\mathrm{x}}^{\prime}+\mathrm{A} \Psi_{\mathrm{y}}^{2}$ A exactly reproduces $\Sigma_{\mathrm{x}}$.

\section{ESTIMATION OF THE}

IPSATIVE COMMON FACTOR MODEL

The common factor model defined above for a set of ipsative variables bears an interesting relationship to the common factor model for the corresponding set of preipsative variables. First, it should be pointed out that the factors of the model remain the same after an ipsative transformation, and the factor covariance matrix remains unchanged. Second, under the ipsative transformation, the factor pattern coefficient matrix contains deviations from the average factor pattern coefficients in the columns of the original factor pattern matrix. Third, the ipsative transformation causes the disturbances to be correlated. These observations have implications for the factor analysis of sets of ipsative variables, and on this basis we suggest that the naive exploratory factor analysis of ipsative variables is inappropriate. Such an analysis would assume that $\mathbf{A}=\mathbf{I}$, and would therefore represent some type of an approximation. The estimated factor pattern in such an analysis would approximate $\left(\wedge_{y}-1 \bar{\lambda}^{\prime}\right)$, however, not $\wedge_{y}$, although the rank-ordering of the magnitudes of the coefficients in the two coefficient matrices would be identical. In any event, there exists a problem with the interpretation of the ipsative factor pattern coefficient matrix since the values in $\bar{\lambda}$ are unknown.

As we indicated above, $\Sigma_{x}$ is singular, and it is not feasible to analyze this matrix without deleting a row and column associated with an arbitrarily selected variable in $x$. Let $x^{*}$ be this set of $p-1$ variables. For present purposes, suppose we have deleted the $\mathrm{p}^{\text {th }}$ variable, although the deletion of any variable 
in this vector will suffice. In fact, the parameters of the ipsative common factor model are invariant regardless of the variable deleted.

We may now write a common factor model for $\mathrm{x}^{*}$ as follows:

$$
\mathrm{x}^{*}=\left[\mathrm{B}_{1} \mid \mathrm{B}_{2}\right]\left[\begin{array}{l}
\xi \\
\epsilon
\end{array}\right]
$$

where $B_{1}$ contains the first $p-1$ rows of the matrix $\left(\Lambda_{y}-1 \bar{\lambda}^{\prime}\right)$ and $B_{2}$ contains the first $p-1$ rows of $A$. Note that $B_{1}$ is a $(p-1) \times k$ submatrix of $\Lambda_{x}^{*}$ and $B_{2}$ is a $(p-1) \times p$ submatrix. In other words $\Lambda_{x}^{*}=\left[B_{1} \mid B_{2}\right]$ is a $(p-1) \times(k+p)$ coefficient matrix. The covariance matrix for $\left[\begin{array}{l}\xi \\ \epsilon\end{array}\right]$ in this case is as follows:

$$
\Phi_{\mathrm{x}^{*}}=\left[\begin{array}{c|c}
\Phi_{\mathrm{y}} & 0 \\
\hline 0 & \Psi_{\mathrm{y}}^{2}
\end{array}\right]
$$

Recall that $\Psi_{y}^{2}$ is diagonal, but $\Phi_{y}$ may be nondiagonal.

Note that the coefficients in in $B_{2}$ are known, so they are to be constrained in the estimation of the model. Our objective, then, is to estimate the parameters in $B_{1}, \Phi_{y}$, and $\Psi_{y}^{2}$, and they may be estimated if the model is identified. A necessary condition for the identification of the model is a minimum of $\mathrm{k}^{2}$ independent constraints on $B_{1}$ and $\Phi_{y}$ (see Jöreskog, 1978; Sörbom and Joreskog, 1976). Finally, we should note that the factor pattern coefficients for the $\mathrm{p}^{\text {th }}$ variable may be obtained by taking the negative of the sum of the first $(p-1)$ values in each column of $B_{1}$.

Using the above illustration to demonstrate the manner by which the coefficients of the ipsative common factor model may be estimated, we present the $k=6$ factor model that would be 
estimated for $\mathrm{x}$ in the above 5 variable cases. The model is as follows:

$$
\begin{aligned}
\Lambda_{x^{*}}= & {\left[\begin{array}{rrrrrr}
-.20 & .80^{\mathrm{f}} & -.20^{\mathrm{f}} & -.20^{\mathrm{f}} & -.20^{\mathrm{f}} & -.20^{\mathrm{f}} \\
-.10 & -.20^{\mathrm{f}} & .80^{\mathrm{f}} & -.20^{\mathrm{f}} & -.20^{\mathrm{f}} & -.20^{\mathrm{f}} \\
.00 & -.20^{\mathrm{f}} & -.20^{\mathrm{f}} & .80^{\mathrm{f}} & -.20^{\mathrm{f}} & -.20^{\mathrm{f}} \\
.10 & -.20^{\mathrm{f}} & -.20^{\mathrm{f}} & -.20^{\mathrm{f}} & .80^{\mathrm{f}} & -.20^{\mathrm{f}}
\end{array}\right] } \\
\Phi_{x^{*}}= & {\left[\begin{array}{cccccc}
2.0 & 0.0^{\mathrm{f}} & 0.0^{\mathrm{f}} & 0.0^{\mathrm{f}} & 0.0^{\mathrm{f}} & 0.0^{\mathrm{f}} \\
0.0^{\mathrm{f}} & .90 & 0.0^{\mathrm{f}} & 0.0^{\mathrm{f}} & 0.0^{\mathrm{f}} & 0.0^{\mathrm{f}} \\
0.0^{\mathrm{f}} & 0.0^{\mathrm{f}} & 1.2 & 0.0^{\mathrm{f}} & 0.0^{\mathrm{f}} & 0.0^{\mathrm{f}} \\
0.0^{\mathrm{f}} & 0.0^{\mathrm{f}} & 0.0^{\mathrm{f}} & 1.0 & 0.0^{\mathrm{f}} & 0.0^{\mathrm{f}} \\
0.0^{\mathrm{f}} & 0.0^{\mathrm{f}} & 0.0^{\mathrm{f}} & 0.0^{\mathrm{f}} & .80 & 0.0^{\mathrm{f}} \\
0.0^{\mathrm{f}} & 0.0^{\mathrm{f}} & 0.0^{\mathrm{f}} & 0.0^{\mathrm{f}} & 0.0^{\mathrm{f}} & .60
\end{array}\right] }
\end{aligned}
$$

Note that the coefficients in the first column of $\wedge_{x} *$ represent deviations of the $\lambda_{i}$ values from the average $\lambda_{i}$ value in the common factor model given above $(\bar{\lambda}=1.2)$. The factor pattern coefficient for the $p^{\text {th }}$ variable is the negative of the sum of the $p-1$ values in the first column of $\wedge_{x} *$, or .20 . The remainder of the $\Lambda_{\mathrm{x}} *$ matrix is constrained equal to the values there (as indicated by the " $f$ " superscript). Note that the $\phi_{11}$ in the $\Phi_{x} *$ matrix equals $\Phi_{y}$, and $\phi_{22}, \phi_{33}, \phi_{44}, \phi_{55}$, and $\phi_{66}$ in $\Phi_{x} *$ are the values of the residual variances for the y vector in $\Psi_{y}^{2}$.

We began this illustration by defining a common factor model for $y$. Since the common factor model for the ipsative set of variables, $x$, is defined, given knowledge of the common factor model for $y$, the above model for $x^{*}$ exactly reproduces the first $(p-1)$ rows and columns of $\Sigma_{x} *$

\section{AN APPLICATION OF THE}

IPSATIVE COMMON FACTOR MODEL

Here we will develop a set of assumptions that permit the application of the above ipsative common factor model to other 
sets of variables that have the ipsative property. We make three critical assumptions: (1) that for a given set of variables with the ipsative property, e.g. a set of rankings, there exists a corresponding set of hypothetical nonipsative variables in the population; (2) that the set of ipsative variables observed in the population of interest is an ipsative transformation of the hypothetical set of nonipsative variables; and (3) that a common factor model holds in the population of interest for the hypothetical set of nonipsative variables.

Given these assumptions, we may estimate the parameters of an ipsative common factor model for any set of variables with the ipsative property. Unfortunately, there is no direct way of determining the veracity of these assumptions for the types of ipsative variables of interest, e.g. rankings, because they have not explicitly undergone an ipsative transformation. We can indirectly assess the adequacy of these assumptions using sample data from sufficiently large samples where the sample moments differ trivially from the population moments. In such cases the failure of our estimated model to reproduce the sample covariance matrix would provide some suggestion about the adequacy of the model. There is a degree of ambiguity here, however, as the failure of the model to reproduce the sample covariances may result from at least two sources: (1) the misspecification of the factor model for the hypothetical $y$, especially the number of factors, and (2) the misspecification of the response process; in other words, errors in the assumptions indicated above for applying the ipsative common factor model. Because there is no known exploratory factor analysis method that can be used to determine an adequate number of factors in the ipsative factor model in this case, there may be some difficulty in specifying the number of factors appropriate for the model.

In determining the goodness-of-fit of a particular application of the ipsative common factor model, the model should be compared with a baseline model that posits no common factors, as in assessing the relative fit of factor models generally. In the present case one would compare a particular model with the following model:

$$
\Sigma_{\mathrm{x}}=\Psi_{\mathrm{x}}^{2}=\mathrm{A} \Psi_{\mathrm{y}}^{2} \mathrm{~A}
$$


The $\chi^{2}$ goodness-of-fit statistic for this model can be used in the computation of a Tucker-Lewis coefficient of relative fit as a baseline model against which the fit of other models may be judged (Tucker and Lewis, 1973).

There are additional approaches to determining the adequacy of the above assumptions for modeling the latent content of sets of variables with the ipsative property which have not explicitly undergone an ipsative transformation. The first of these involves a systematic study of the response process involved in the production of such variables. What is the relation between the response process involved in ranking $p$ objects and the response process involved in obtaining nonipsative measurements-for example, ratings of $p$ objects along some dimension? Can the relation be described by a mathematical function such $\mathrm{x}=\mathrm{Ay}$ ? We will not pursue this set of issues here except to note that little is known about the relationship. A second approach to assessing the above assumptions for such ipsative measures involves Monte Carlo simulation. Here it is possible to compare the factors that result from making different sets of assumptions regarding the response process.

\section{AN ANALYSIS OF \\ KOHN'S MEASURES OF PARENTAL VALUES}

In this section of the article we apply the ipsative common factor model to Kohn's measures of parental desires for children's behavior (Kohn, 1969: 257). Kohn developed the following set of questions for measuring parental child-rearing values:

(a) Which three qualities listed on this card would you say are the most desirable for a (boy, girl) of (child's) age to have?

(b) Which one of these three is the most desirable of all?

(c) All of these may be desirable, but could you tell me which three you consider least important?

(d) And which one of these three is least important of all?

(1) that he has good manners

(2) that he tries hard to succeed

(3) that he is honest

(4) that he is neat and clean 
(5) that he has good sense and sound judgment

(6) that he has self-control

(7) that he acts like a boy (she acts like a girl) should

(8) that he gets along well with other children

(9) that he obeys his parents well

(10) that he is responsible

(11) that he is considerate of others

(12) that he is interested in how and why things happen

(13) that he is a good student.

Using this information Kohn (1969: 48) coded the responses according to the following scheme:

$5=$ The most valued of all.

$4=$ One of the three most valued, but not the most valued.

$3=$ Neither one of the three most nor one of the three least valued.

$2=$ One of the three least valued, but not the least valued.

$1=$ The least valued of all.

This measurement strategy represents a reduced-ranking procedure. Instead of ranking the 13 characteristics from most to least important, respondents are essentially asked to sort the characteristics into 5 ranked categories with a requisite number in each category. In either case, such a ranking procedure produces a set of scores with the ipsative property.

In order to use these data to relate parental values to social class, Kohn (1969: 56-58) employed the methods of exploratory factor analysis to form a weighted composite from these items. Some weighting of the item scores was obviously necessary, as the unweighted sum of the items has no variability over individuals. He reported some difficulty in inverting the correlation matrix among his measures for the purpose of computing factor scores, which would be predicted given knowledge of the ipsative property of the measures. In any event, according to Kohn (1969: 57), the exploratory factor analysis of these measures "yields one factor easily identified as self-direction versus conformity to external standards" and two additional factors. He focused his primary attention, however, on the self-direction/ conformity factor. 
For our purposes, we will use sample data on Kohn's measures from the General Social Surveys (GSS) of 1973, 1975, 1976 and 1978 (see Alwin and Jackson, 1980, for a complete description of the population of interest and the precise nature of the measures used in the GSS). Table 1 presents the sample covariance matrices for the subpopulations of mothers $(N=1,069)$ and fathers $(\mathrm{N}=721)$ from the NORC-GSS surveys. The sample data from the subpopulations of mothers and fathers are analyzed separately because of the expectation of substantive differences in the coefficients of the factor model. As noted above, the rows (or columns) of the full symmetric covariance matrices for these variables sum to zero, a property of ipsative covariance matrices.

In this analysis we obtain maximum-likelihood estimates of the unconstrained parameters of the ipsative common factor model developed above (see equations 6 and 7) using confirmatory factor analysis (Sörbom and Jöreskog, 1976). The results for this model where $k=1$ are given in Tables 2 and 3 . We have identified the model by setting $\Phi_{y}$ (or $\phi_{\xi}$ ) equal to unity, and because $A$ and $\Phi_{y}$ contain only fixed parameters, we have estimated the 26 parameters in $\Lambda_{x}$ and $\Psi_{y}^{2}$. The estimated coefficients of $\Lambda_{x}$ are deviations of the factor pattern coefficients of $\Lambda_{y}$ from their average, $\bar{\lambda}$. The value of $\bar{\lambda}$ is, however, unknown, but this is irrelevant, since whatever its value, the coefficients in $\Lambda_{x}$ maintain the same relative magnitude.

The relative rankings of the pattern coefficients in the estimated $\Lambda_{\mathrm{x}}$ in Tables 2 and 3 conform to our substantive expectations, and these results show considerable similarity for mothers and fathers. The measures indicating a preference for self-directed behavior in children (items 12, 5, 10, 11,6) covary in one direction with the factor, while the measures indicating conformity to external authority (items $9,1,4,3,7$ ) covary in the opposite direction with the factor. Because the coefficients in $\wedge_{x}$ are identified up to a change in sign, the direction of the latent factor is dependent on the starting values.

The pattern of coefficients reported in Tables 2 and 3 are similar, although not identical, to the results reported by Kohn for his original data and his reanalysis of the 1973 GSS data (Kohn, 1976: 540). In terms of the pattern of coefficients ob- 


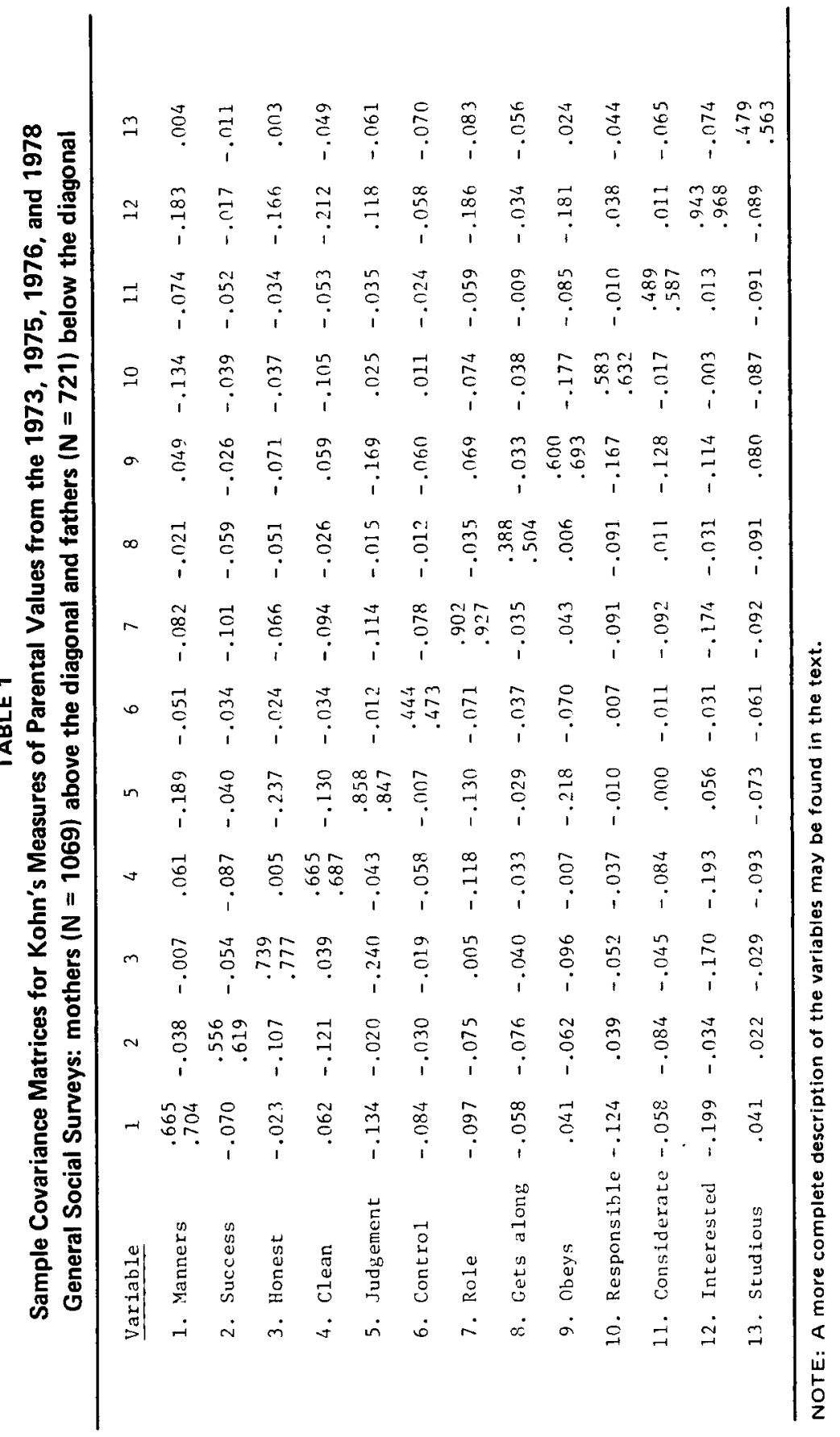




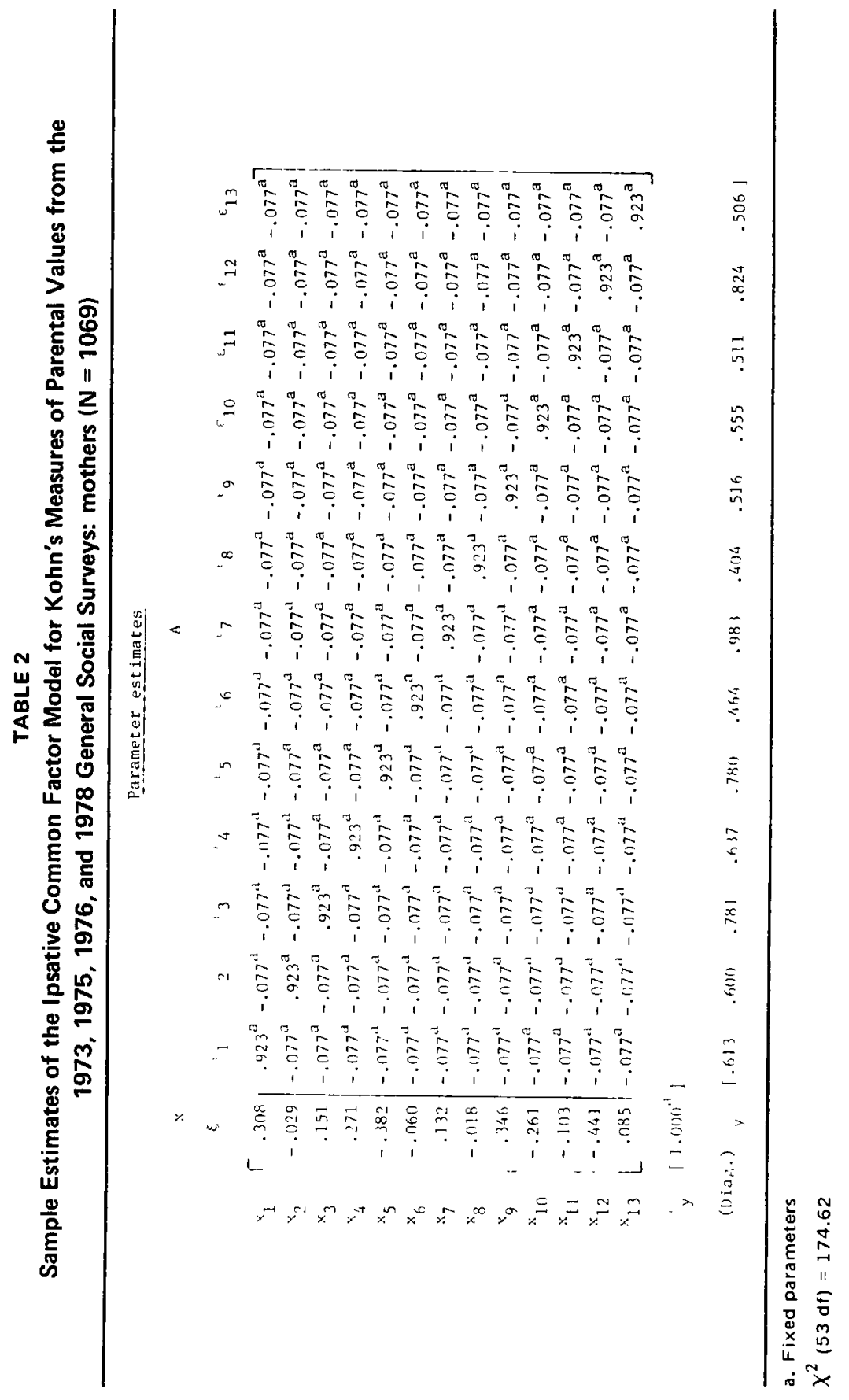




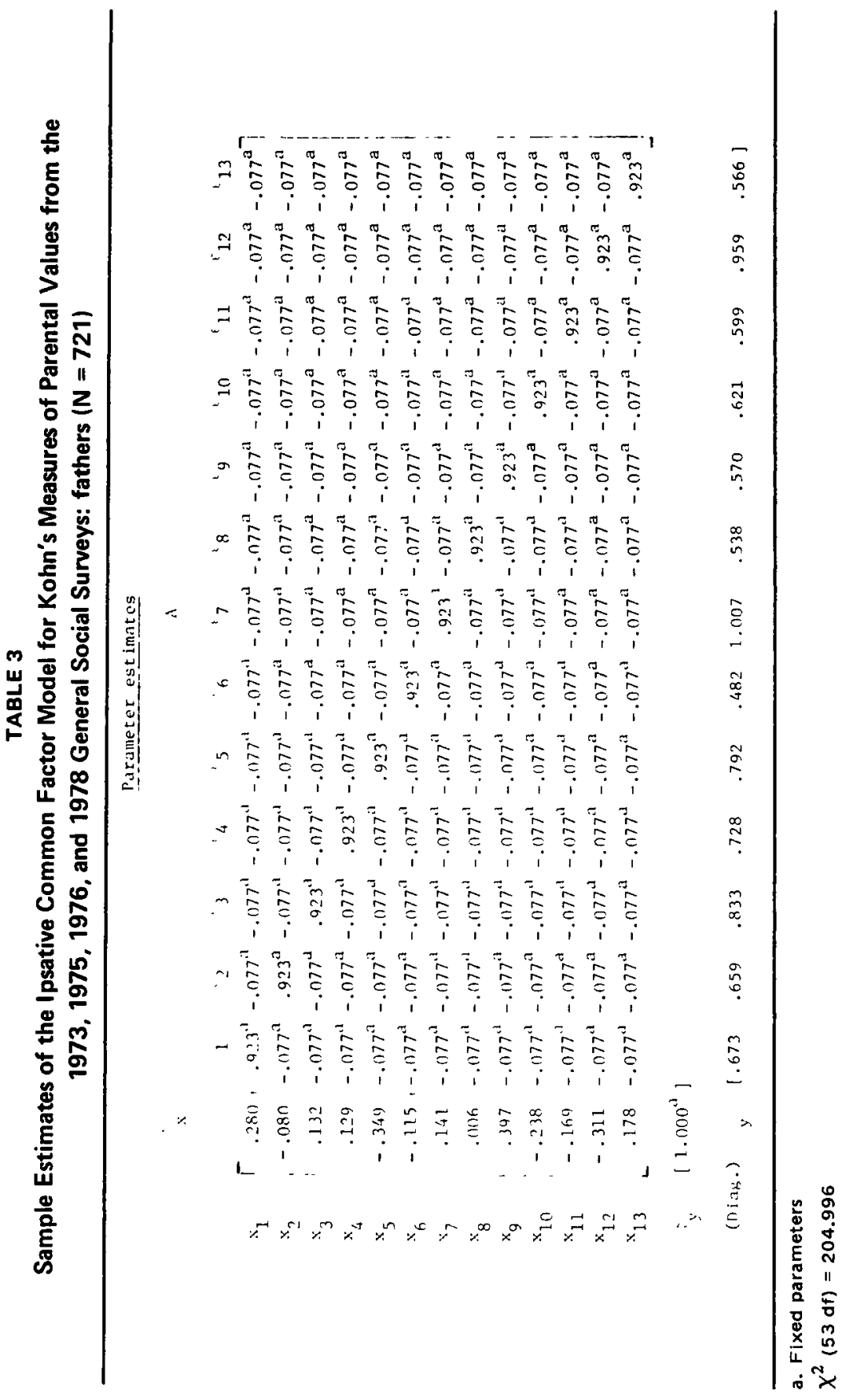


tained, the approximation to the ipsative common factor model using the methods of exploratory factor analysis in this case appears to be relatively good, although the extent to which these results can be generalized is unclear. As we pointed out in the above discussion, as $p$ increases, the ipsative transformation matrix approaches an identity. The consequence of this is to reduce the effects of the ipsative transformation on the covariance structure. In any event, it is not clear to us how large $p$ must be to safely ignore the effects of the ipsative property of the measures. We are reluctant to conclude from the present findings that the ipsative property of these parental values indicators may safely be ignored.

As we indicated in the preceding discussion, it is necessary to consider the overall fit of the ipsative common factor model in order to evaluate its utility. In Tables 2 and 3 we have reported the likelihood-ratio $\chi^{2}$ values for evaluating the goodness-of-fit of the model presented there. Under the assumption of multivariate normality in the $y$ vector, these $\chi^{2}$ values may be used as measures of fit in large samples (see Jöreskog, 1978: 447). The $\chi^{2}$ values obtained both for mothers $\left(\chi^{2}=174.62\right)$ and fathers $\left(\chi^{2}=204.99\right)$ with 53 degrees of freedom are statistically significant at extremely low levels of Type I error, and provide a statistical basis for the rejection of the model. It is important, however, to evaluate the goodness-of-fit of this model in light of the fit of alternative models. This is partly due to the fact that the above likelihood-ratio $\chi^{2}$ statistics are partially a function of sample size and cannot therefore be interpreted unambiguously in large samples (Jöreskog, 1978: 447).

In addition, statistical measures of fit generally have meaning only within a comparative framework, and it is useful therefore propose meaningful alternatives to the present model (Jöreskog, 1978: 448). Therefore, as suggested above, we compare the results of the model presented in Tables 2 and 3 with the results of a model that posits no latent common factors for the variables; i.e., $\Sigma_{x}=\Psi_{x}^{2}=$ A $\Psi_{y}^{2}$ A. In addition, we examine a model that posits two latent factors for the observed indicators.

The model suggested here as a baseline against which to compare the present model provides a poorer fit to the sample 
data for both mothers $\left(\chi^{2}=475.05\right)$ and fathers $\left(\chi^{2}=356.59\right)$, as would be expected. Using the Tucker-Lewis coefficient (TLC) to assess the relative fit of our model over this zero-factor model, a slight improvement is revealed for fathers (TLC $=.361$ ) and a moderate improvement is revealed for mothers (TLC $=.636$ ). In other words, within the set of assumptions we have made here in order to apply the ipsative common factor model to this particular set of ipsative variables, there is evidence that a selfdirection/conformity factor, originally identified by Kohn (1969: 57), exists for these measures. At the same time, the evidence for this is not strong, in the sense that the model allowing for this latent factor provides only a modest improvement in fit to the sample data over a model that posits no such factor.

As noted in the above discussion, there is no direct way within the present context to evaluate the adequacy of the set of assumptions we have made in order to apply the ipsative common factor model in the present case. The relatively poor fit of our model to the sample data used here may indicate problems with this particular set of assumptions or with the complexity of the factor model assumed to hold for the y vector. In order to examine this second possibility we have estimated a 2-factor ipsative common factor model. The results for this model reflect an improved fit to the data for both mothers $\left(\chi^{2}=99.33\right.$, TLC $\left.=.792\right)$ and fathers $\left(\chi^{2}=133.89\right.$, TLC $\left.=.529\right)$, but the overall fit to the data in either case, as reflected in the Tucker-Lewis coefficients, is not entirely satisfactory. We suspect that the addition of factors to the model will improve the overall fit to the sample data, but it is unclear whether this would add substantially to our understanding of the applicability of the ipsative common factor model to these measures.

\section{SUMMARY AND CONCLUSIONS}

In the above presentation we have developed a common factor model for variables that have undergone an ipsative transformation, given knowledge of the factor model for the preipsative variables. The ipsative common factor model has been 
defined, given the existence of two facts: (1) $x=A y$ and (2) $y=$ $\wedge_{y} \xi+\epsilon$. In the remainder of the article we have addressed the question of the applicability of the ipsative common factor model to sets of variables with ipsative properties which result from the measurement procedure rather than an ipsative transformation of nonipsative variables. We argued that if one is willing to assume that there exists a hypothetical nonipsative set of measures $(y)$ corresponding to the ipsative set $(x)$, that $x=A y$, and that $y=\wedge_{y} \xi+\epsilon$, then it is possible to apply the ipsative common factor model to such measures. Unfortunately, there is no direct way to evaluate the measures' validity within this set of assumptions.

The evaluation of the applicability of the ipsative common factor model in these cases requires (1) an examination of the differences in the response processes involved in producing data with ipsative and nonipsative properties, e.g. rankings vs. ratings, and (2) the specification of plausible alternative models for sets of ipsative variables that represent both the latent content of the variables and their ipsative properties. Finally, there is also some utility in applying the ipsative common factor model to sets of ipsative variables, such as Kohn's measures of parental values, in order to obtain greater experience regarding the descriptive usefulness of the ipsative common factor model.

\section{NOTES}

1. Horst (1965: 287-288) refers to the ipsative transformation as the "minor transformation" or "right centering," while the normative transformation is called the "major transformation" or "left centering." When both transformations are performed, the scores are said to be "double-centered." See also Harris (1953), Tucker (1956), and Hicks (1970).

2. Horst (1965: 292) shows that ipsative measures that sum to the constant $\mathrm{c}$ for each individual will sum to zero when the $x$ 's are left-centered.

3. This is a function of the fact that as p increases, the ipsative transformation matrix, A, approaches an identity.

4. This observation may not apply to all methods of factor analysis, but methods that utilize generalized least squares (GLS) and maximum likelihood (ML) procedures to fit the model require a positive-definite sample covariance matrix (Jöreskog, 1978: 446). 


\section{REFERENCES}

AL WIN, D. F. and D. J. JACKSON (1980) "The statistical analysis of Kohn's measures of parental values." In H.O.A. Wold and K. G. Joreskog (eds.) Systems Under Indirect Observation: Causality, Structure and Prediction. New York: Elsevier NorthHolland.

CATTELL, R. B. (1952) Factor Analysis. New York: Harper \& Row.

- (1944) "Psychological measurement: ipsative, normative and interactive." Psych. Rev. 51: 292-303.

CLEMANS, W. V. (1956) "An analytical and empirical examination of some properties of ipsative measures." Ph.D. dissertation, University of Washington.

CUNNINGHAM, W. H., I.C.M. CUNNINGHAM, and R. T. GREEN (1977) "The ipsative process to reduce response set bias." Public Opinion Q. 41: 379-384.

GUILFORD, J. P. (1954) Psychometric Methods. New York: McGraw-Hill.

HAR RIS, C. W. (1953) "Relations among factors of raw, deviation and double-centered score matrices." J. of Experimental Education 22: 53-58.

HICKS, L. E. (1970) "Some properties of ipsative, nor mative and forced choice normative measures." Psych. Bull. 74: 167-184.

HORST, P. (1965) Factor Analysis of Data Matrices. New York: Holt, Rinehart \& Winston.

JORESKOG, K. G. (1978) "Structural analysis of covariance and correlation matrices." Psychometrika 43: 443-477.

KOHN, M. L. (1976) "Social class and parental values: another confirmation of the relationship." Amer. Soc. Rev. 41: 538-545.

_- (1969) Class and Conformity: A Study in Values. Homewood, IL: Irwin.

LAWLEY, D. N. and A. E. MAXWELL (1971) Factor Analysis as a Statistical Method. New York: Elsevier North-Holland.

SORBOM, D. and K. G. JORESKOG (1976) COFAMM-Confirmatory Factor Analysis with Model Modification. Chicago: National Educational Resources.

TUCKER, L. R. (1956) "Factor analysis of double-centered score matrices." Research Memorandum (RM-56-3). Princeton, NJ: Educational Testing Service.

_analysis." Psychometrika 38: 1-10.

David J. Jackson is a research sociologist for the Population Research Section of the Mental Health Study Center, National Institute of Mental Health. His research interests are factorial ecology, use of census data in mental health-related research. and measurement.

Duane F. Alwin is Associate Research Scientist in the Survey Research Center and Associate Professor in Sociology at The University of Michigan. In addition 10 the quantitative analysis of measurement issues, his research interests include the sociology of education and the social psychology of inequality. 